\title{
Granulomatosis with Polyangiitis with Intestinal Perforations as Complications: Case Report
}

\author{
Cobos Quevedo Orestes de Jesús*1 and Ramírez Montelongo Sandra María \\ ${ }^{1}$ Department of Internal Medicine, Mexico
}

${ }^{2}$ Head of Internal Medicine Service, Mexico

Received: 眥: December 13, 2018; Published: 帮: January 04, 2019

*Corresponding author: Cobos Quevedo Orestes de Jesús, Department of Internal Medicine, IMSS(Mexican Institute of Social Security), Zone 27 General Hospital, Mexico

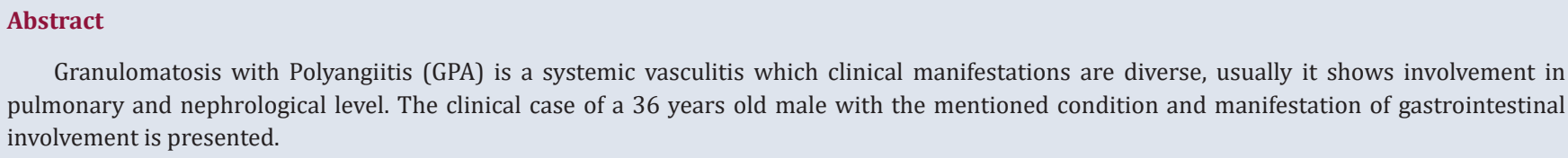

Granulomatosis with Polyangiitis (GPA) is a systemic vasculitis which clinical manifestations are diverse, usually it shows involvement in pulmonary and nephrological level. The clinical case of a 36 years old male with the mentioned condition and manifestation of gastrointestinal involvement is presented.

Keywords: Case Report; Granulomatosis with Polyangiitis; Intestinal Perforation; Vasculitis

\section{Introduction}

Granulomatosis with polyangiitis (previously known as Wegener's Granulomatosis) is a necrotizing vasculitis of small blood vessels with multiple system impact characterized by necrosis and granulomatous inflammation, it was described for the first time by Klinger in 1931 and later by Wegener in 1936 [1]. It is a systemic clinical-pathological entity mediated by immune mechanisms. The most common clinical manifestations are of otorhinolaryngological, respiratory and renal origin. The gastrointestinal involvement is rare and a poor prognosis marker [2,3]. The clinical case of a patient with granulomatosis with polyangiitis, intestinal perforation and secondary peritonitis is presented in Figure 1.

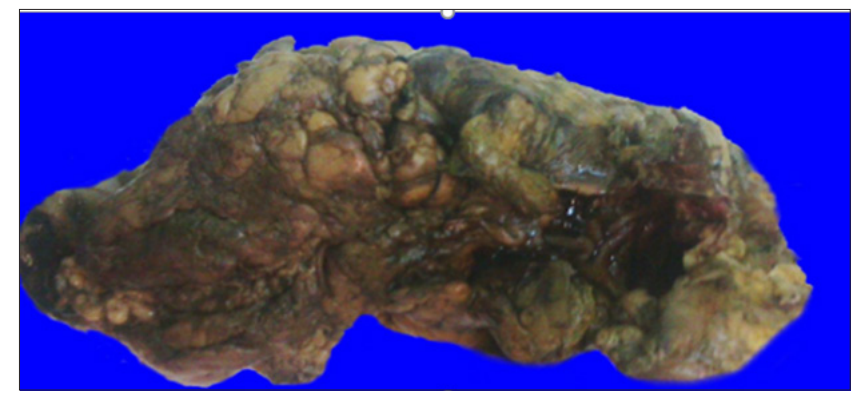

Figure 1: Final histopathological diagnosis: Intestinal peroration with transluminal enteritis and acute peritonitis.

\section{Case Presentation}

A 36 years old male without serious pathological elements in his past history presents a clinical picture of one-month evolution with arthralgia, fever, dyspnea on medium exertion associated with hemoptysis and weight loss of 7 kilograms. He goes into hospitalization, in his assessment, purpuric injuries are found on the skin, renal failure, anemia, leukocytosis and positive tests for c-ANCA antibodies are documented. In the computed tomography of the chest hyper dense paramilitary objects and ground glass areas are observed. The bronchoscopy shows diffuse alveolar bleeding and inflammatory changes. Kidney biopsy with proliferative extra capillary glomerulonephritis with fibrinoid necrosis. He is given 1 gram of methylprednisolone per day in 3 doses and in the 4th day, 1 gram of cyclophosphamide is added. 24 hours later he develops a septic shock. A laparotomy is performed, and colonic perforation is found, showing torpid post operatory evolution with eventual death. Final histopathological diagnosis: Intestinal perforation with transluminal enteritis and acute peritonitis.

\section{Discussion}

The patient in the case was diagnosed with granulomatosis with polyangiitis based on clinical criteria, positive tests for c-ANCA and kidney biopsy consistent with vasculitis of small blood vessels. The literature documents gastrointestinal involvement is rare, nevertheless it is the cause of death IN $12 \%$ of the cases of GPA, 
in recent reports the gastrointestinal illness includes: submucosal edema, ulcers, hemorrhage, mesenteric ischemia, intestinal obstruction and perforation [4-6]. Colonic perforation associated with cytomegalovirus infection in immunocompromised patients (kidney transplant, systemic erythematosus lupus, HIV infection) precipitated by treatment with steroids has been documented in literature, however literature regarding GPA has not been found [7]. In patients with perforation on the left side of the colon, it may be difficult to distinguish between diverticulitis precipitated by vasculitis $o$ as a complication of the immunosuppressive therapy, or a combination of both. The use of immunosuppressants, mainly steroids in high doses has been associated as the etiology of intestinal necrosis and the perforation, this hypothesis is backed up by the absence of vasculitis in the histopathological report of our case even though the treatment could also alter the histopathological results. On the other hand, spontaneous perforation even without treatment has been observed and also healing of the intestinal perforation after the administration of immunosuppressants has been documented, besides, a successful treatment with rituximab in patients that presented intestinal perforation as complication has been observed [3,7-9].

\section{Conclusion}

The gastrointestinal involving, as the case of perforation is rare, but relevant among the complications, its handling merits close cooperation between surgeons and rheumatologists. This complication may be related with the immunosuppressive treatment although its novo appearance with good response to the treatment has also been documented, despite it, the prognosis is poor due to the high mortality rate according to the reported cases.

\section{References}

1. Greco A, Marinelli C, Fusconi M, Macri G, Gallo A, et al. (2015) Clinic manifestations in granulomatosis with polyangiitis. Int J Immunopathol Pharmacol 29(2): 151-159.

2. Pagnoux C, Guillevin L (2015) Treatment of granulomatosis with polyangiitis (Wegener's). Expert Rev Clin Immunol 11(3): 339-348.

3. Kiboshi T, Isoda K, Furukawa K, Wakahara T, Otani K, et al. (2017) Granulomatosis with polyangiitis complicated with gastrointestinal perforation: A case report and review of literature. Nihon Rinsho Meneki Gakkai Kaishi 40(5): 382-386.

4. Toh JWT, Fehlberg T, Raashed S, Patapanian H, Turner C (2018) Unexpected bowel perforation in Wegener's granulomatosis. ANZ J Surg 88(3): 245-247.

5. Pan SW, Wang C, Zhang X, Zhang L, Yan QQ, et al. (2018) A rare endoscopic appearance of granulomatosis with polyangiitis involving the intestine: A case report. BMC Gastroenterol 18(1): 154.

6. Dinic M, Kandolf SL, Zolotarevski L, Zecevic R (2013) Fulminant Wegener's granulomatosis: A case report. Vojnosanit Pregl 70(9): 887890.

7. Soriano A, Smerieri N, Bonilauri S, De Marco L, Cavazza A, et al (2018) Colonic perforation due to severe cytomegalovirus disease in granulomatosis with polyangiitis after immunosuppression. Clin Rheumatol 37(5): 1427-1432.

8. Ikeda K, Takasaki Y, Sekigawa I (2014) Rapid onset of small intestinal perforation after successful steroid treatment in eosinophilic granulomatosis with polyangiitis. Mod Rheumatol 26(6): 1-3.

9. Dag MS, Pehlivan Y, Tutar E (2013) Rituximab seems a promising therapeutic option in granulomatosis with polyangiitis with intestinal perforation: A case report and literature review. BMJ Case Reports.
ISSN: 2574-1241

DOI: 10.26717/BJSTR.2019.12.002309

Cobos Quevedo Orestes de Jesús. Biomed J Sci \& Tech Res

(c) (P) This work is licensed under Creative

Submission Link: https://biomedres.us/submit-manuscript.php

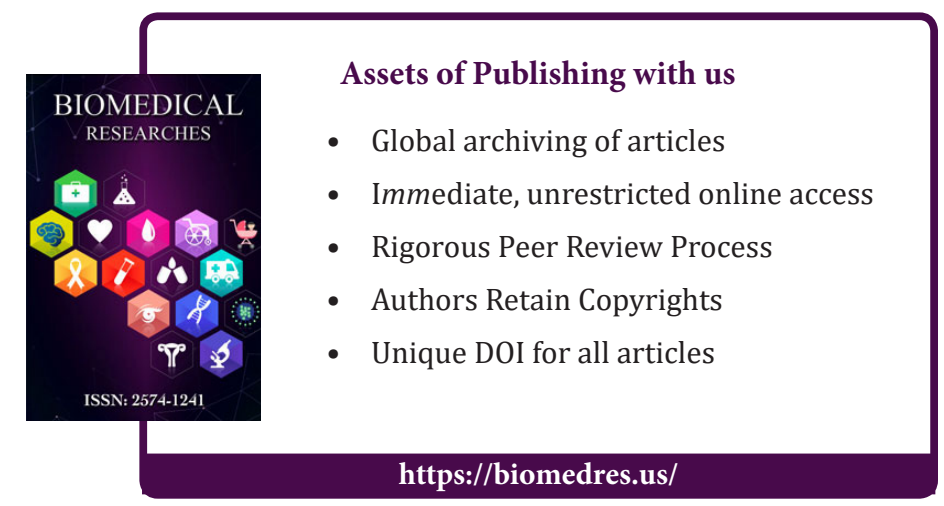

